Note

\title{
QTL analysis for brown spot resistance in American rice cultivar 'Dawn'
}

\author{
Yuya Ota ${ }^{1)}$, Kengo Matsumoto'), Yukinori Nakayama ${ }^{1)}$, Tomohiro Yamakawa'), Teppei Ohno'), \\ Ritsuko Mizobuchi*3) and Hiroyuki Sato*4) \\ 1) Mie Prefecture Agricultural Research Institute, 530 Ureshinokawakita, Matsusaka, Mie 515-2316, Japan \\ 2) Kuwana Agricultural Forestry Fisheries Office, Kuwana, Mie 511-8567, Japan \\ 3) Institute of Crop Science, National Agriculture and Food Research Organization (NARO), 2-1-2 Kannondai, Tsukuba, Ibaraki \\ 305-8602, Japan \\ 4) Ministry of Agriculture, Forestry and Fisheries, 1-2-1 Kasumigaseki, Chiyoda-ku, Tokyo 100-8950, Japan
}

Rice brown spot (BS), caused by Bipolaris oryzae, causes yield loss and deterioration of grain quality. Using single-nucleotide polymorphism (SNP) markers, we conducted quantitative trait locus (QTL) analysis of BS resistance in backcross inbred lines (BILs) from a cross between an American rice cultivar, 'Dawn' (resistant), and 'Koshihikari' (susceptible). Four QTLs for BS resistance were detected in a three-year field evaluation, and 'Dawn' contributed the resistance alleles at all QTLs. The QTL with the greatest effect, $q B S R 6-k d$, explained $15.1 \%$ to $20.3 \%$ of the total phenotypic variation. Although disease score and days to heading (DTH) were negatively correlated in all three years, $q B S R 6-k d$ was located near a QTL for DTH at which the 'Dawn' allele promoted heading. Another BS resistance QTL $(q B S R 3.1-k d)$ was unlinked to the QTLs for DTH. Therefore, these two QTLs are likely to be useful for breeding BS-resistant varieties without delaying heading. The other two BS resistance QTLs ( $q B S R 3.2-k d$ and $q B S R 7-k d)$ were located near DTH QTLs at which the 'Dawn' alleles delayed heading. The QTLs reported here will be good candidates for developing BS-resistant cultivars.

Key Words: Bipolaris oryzae, brown spot, heading date, Oryza sativa L., QTL analysis, resistance, rice.

\section{Introduction}

Rice brown spot (BS), caused by the fungal pathogen Bipolaris oryzae, is one of the most serious diseases of rice worldwide. The fungus can infect rice plants from the young seedling stage to the mature stage and causes significant yield loss (Sunder et al. 2014). Spread of the disease was the main cause of the Great Bengal Famine (Padmanabhan 1973). In Japan, the epidemic area of BS in 2018 was the third largest for any rice pathogen (197,187 ha), after those of sheath blight (539,641 ha), and rice blast (296,518 ha) (Japan Plant Protection Association 2019).

Recent expansion of the BS endemic area in Japan was caused by two factors. First, the application frequency or doses of fungicides, soil conditioners, and fertilizers have been reduced to promote eco-friendly agriculture (Hori et al. 2019, Yamaguchi et al. 2007). Second, the periods of

Communicated by Qian Qian

Received February 22, 2021. Accepted April 18, 2021.

First Published Online in J-STAGE on August 18, 2021.

*Corresponding authors (e-mail: ritsuko@affrc.go.jp and

bsato@affrc.go.jp) optimal temperature for pathogen growth have been lengthened by climate change (Mizobuchi et al. 2016). Although fungicide application is effective for reducing the disease, the use of genetic resistance is the most practical way because it allows controlling the disease with less fungicide and lower production costs.

Several research groups, including ours, have reported genotypic variability among rice cultivars for BS resistance and have conducted quantitative trait locus (QTL) analyses of BS resistance (Mizobuchi et al. 2016 and references therein, Matsumoto et al. 2017a). Although we have recently detected several QTLs for BS resistance, none of them explained over $30 \%$ of the phenotypic variation in the analysis (Matsumoto et al. 2017b, Sato et al. 2008, 2015). To develop practical cultivars with BS resistance, it will be essential to identify new QTLs from different resistant cultivars for gene pyramiding.

An American rice cultivar, 'Dawn', is highly resistant to BS (Eruotor 1986), and we confirmed its resistance in Japan (Matsumoto et al. 2016). The aim of this study was to detect BS resistance QTLs and develop a BS-resistant line in which a segment of 'Dawn' chromosome would be introgressed into the genetic background of 'Koshihikari', the most popular cultivar in Japan. 


\section{Materials and Methods}

\section{Plant materials}

The BS-resistant donor, Oryza sativa L. 'Dawn' (acc. no. JP14671), was provided by the Gene-Bank Project of the National Agriculture and Food Research Organization (NARO), Tsukuba, Japan. The recurrent parent, O. sativa L. 'Koshihikari', is susceptible to BS. To develop backcross inbred lines (BILs), 'Dawn' was crossed with 'Koshihikari'; then, successive backcrossing with 'Koshihikari' was performed to produce $179 \mathrm{BC}_{2} \mathrm{~F}_{1}$ plants. Generations were advanced by single-seed descent. A population of 179 BILs at the $\mathrm{BC}_{2} \mathrm{~F}_{5}$ generation was used for field evaluation in 2017, 2018, and 2019.

\section{Fungal strain}

Bipolaris oryzae strain iga-2 (MAFF245177) was used as the inoculum. Inoculation, culture of the mycelia, and induction of conidiophore formation under irradiation with black-light lamps were based on the methods of Kihara and Kumagai (1994).

\section{Field evaluation of BS resistance and days to heading (DTH)}

In 2017, 2018, and 2019, BS resistance was evaluated for QTL analysis in a paddy field at Mie Prefecture Agricultural Research Institute (Iga, Mie, Japan) as described by Matsumoto et al. (2016), with two replications per year. To induce BS development, susceptible cultivar 'Mienoyume' inoculated with $B$. oryzae 20 days after sowing by spraying with $40 \mathrm{ml}$ conidial suspension $\left(1 \times 10^{5}\right.$ conidia ml $\left.{ }^{-1}\right)$ was used as a spreader. These spreader plants were planted at a rate of three to five plants per hill, at a spacing of $30 \times 30 \mathrm{~cm}$ among the plants. Then, each BIL and its parents were transplanted between the spreader rows. The average value of two plants for each replicate was used as the disease score. Plant materials were sown and transplanted on May 15 and June 1, respectively, in 2017; May 12 and May 29, respectively, in 2018; and May 9 and May 30 , respectively, in 2019. Disease scores using a scale from 0 (no incidence) to 9 (severe) were recorded on September 14 in 2017, September 12 in 2018, and September 19 in 2019.

In each of the three years, days to heading (DTH) was determined using the average value of two replicates per line. For each replicate, DTH was calculated as the number of days between the date of sowing and the date on which the first panicles had emerged on about $50 \%$ of the individuals (heading date).

\section{Linkage map and QTL analysis}

We extracted total DNA from the leaves of the $\mathrm{BC}_{2} \mathrm{~F}_{5}$ plants and parental cultivars using the CTAB method (Murray and Thompson 1980). To survey the genotypes, single-nucleotide polymorphism (SNP) analysis was per- formed as described by Sato et al. (2015). SNP markers were selected from diverse accessions of cultivated Asian rice (Ebana et al. 2010) and genotyping was performed by using the GoldenGate BeadArray technology platform (Illumina Inc., San Diego, CA, USA). A total of 112 SNP markers showing polymorphism between 'Koshihikari' and 'Dawn' were used to genotype the BILs (Supplemental Table 1). The linkage map was constructed by using version 3.0 of MAPMAKER/EXP (Lander et al. 1987) and visualized by using a Microsoft Excel macro, MapDraw (Liu and Meng 2003). Genetic distances were calculated by the Kosambi function. QTL analysis was performed by using version 2.5 of Windows QTL Cartographer (Wang et al. 2006) with the default composite interval mapping and control parameters, standard model 6 , five control markers, a 10-cM window size, and the forward and backward regression model. The genome-wide threshold value $(\alpha=0.05)$ was used to detect putative QTLs based on the results of 1000 permutations. QTLs were named according to the rice gene nomenclature system (McCouch 2008).

\section{Results}

\section{Phenotypic evaluation of BILs and their parents}

Distinct differences in BS resistance were observed between the parental cultivars, 'Dawn' and 'Koshihikari' (Fig. 1). The mean disease scores of 'Dawn' over the three years were 2.0-2.7, whereas those of 'Koshihikari' were 5.0-5.7 (Fig. 2). The disease scores of the BIL population in each of the three years were normally distributed. Some BILs exhibited transgressive segregation in both directions, further indicating that BS resistance was a quantitative trait (Fig. 2). Significant negative correlations between disease scores and DTH were found in the BIL population in each of the three years $(r=-0.18, P<0.05$ in $2017 ;-0.45$, $P<0.001$ in 2018; and $-0.26, P<0.001$ in 2019). The mean DTH of 'Dawn' (averaged over the three years of the study) was 94, whereas that of 'Koshihikari' was 82 .

\section{QTL analysis for BS resistance}

A linkage map consisting of 12 chromosomes was constructed by genotyping the BILs with 112 SNP polymorphic markers. The percentages of genotypes across all

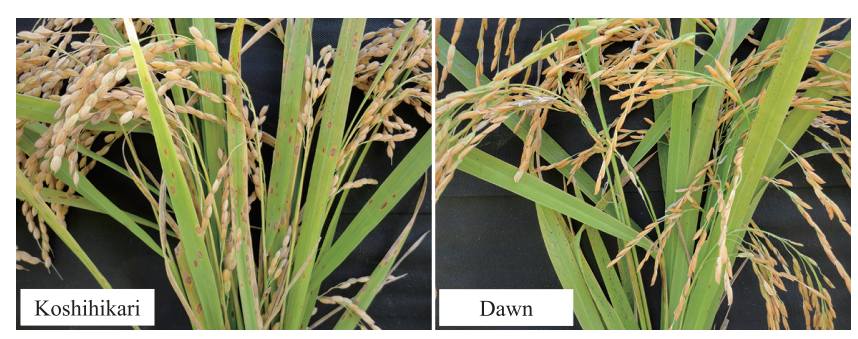

Fig. 1. Brown spot lesions on the leaves of 'Koshihikari' (susceptible) and 'Dawn' (resistant). Photos were taken on September 20, 2019. 


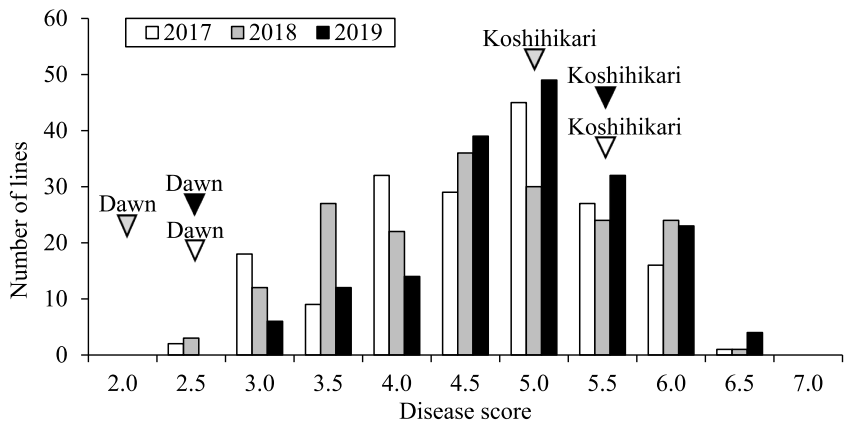

Fig. 2. Frequency distribution of brown spot disease score in 179 BILs (Koshihikari*3/Dawn) in 2017, 2018, and 2019. Arrowheads indicate the mean values for the parents each year. The number below each bin represents its midpoint; for example, bin 2.0 represents trait values from $\geq 1.75$ to $<2.25$.

marker loci in the BIL population were as follows: $84.9 \%$ homozygous for the 'Koshihikari' allele, 10.1\% homozygous for the 'Dawn' allele, $4.3 \%$ heterozygous, and $0.7 \%$ missing data. Excluding the heterozygous regions, the genotypes fit a ratio of 7/8 (homozygous for the 'Koshihikari' allele): $1 / 8$ (homozygous for the 'Dawn' allele), as expected in $\mathrm{a}_{2}$ population. The total length of the map was $428.9 \mathrm{cM}$, with an average distance between adjacent markers of $4.3 \mathrm{cM}$. The genome coverage of the map was $88.5 \%(330.2 \mathrm{Mb} / 373.2 \mathrm{Mb}$ of the $O$. sativa L. 'Nipponbare' pseudomolecule, IRGSP-1.0), as estimated from the physical position of markers at the distal end of each chromosome (Fig. 3).

Four QTLs for BS resistance ( $q B S R 3.1-k d, q B S R 3.2-k d$, $q B S R 6-k d$, and $q B S R 7-k d$ ) were identified based on the data from 2017, 2018, and 2019 (Fig. 3, Table 1). The 'Dawn' alleles explained $6.2 \%-6.8 \%, 7.0 \%, 15.1 \%-20.3 \%$, and $18.6 \%$ of total phenotypic variation, respectively. Two of the QTLs ( $q B S R 3.1-k d$ and $q B S R 6-k d)$ were detected in two years, whereas the other two were each detected in only one year. Among the four QTLs, qBSR6- $k d$ gave the highest percentage of variance explained $(20.3 \%$ in 2017) among the QTLs detected and was considered a major QTL. Since significant negative correlations between disease score and DTH were found in each of the three years, we also mapped QTLs for DTH. Three QTLs for DTH ( $q D T H 3-k d, q D T H 6-k d$, and $q D T H 7-k d$ ) were detected near three of the QTLs for BS resistance (Fig. 3, Table 1). These QTLs were located at the same marker interval in each of the three years. At $q D T H 6-k d$, the QTL allele from 'Dawn' promoted earlier heading, whereas the 'Dawn' alleles at the other two QTLs delayed heading.
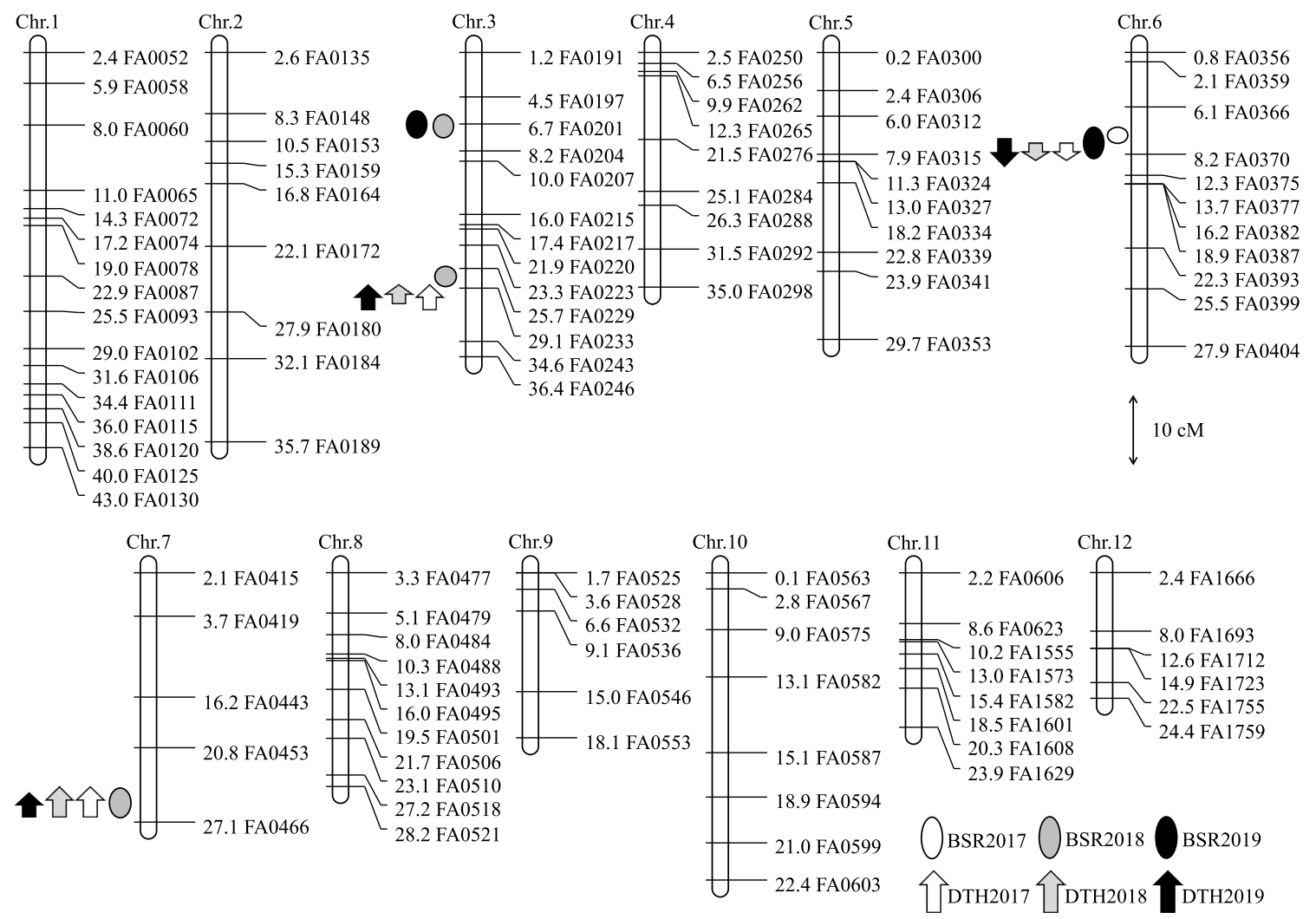

Fig. 3. Genetic linkage map of 112 SNP markers and positions of the QTLs for brown spot (BS) resistance and days to heading (DTH) detected in 179 BILs (Koshihikari*3/Dawn). Positions (Mb) are indicated to the left of the SNP marker names according to the 'Nipponbare' pseudomolecule (IRGSP-1.0). Ovals and arrows represent QTL positions and the size of them means one-LOD confidence intervals for BS resistance and DTH, respectively. Up and down arrows indicate that the 'Dawn' alleles increase and decrease DTH, respectively. 
Table 1. Putative QTLs for brown spot resistance and days to heading

\begin{tabular}{|c|c|c|c|c|c|c|c|c|c|}
\hline Trait & Year(s) & QTL & $\begin{array}{c}\text { Chromo- } \\
\text { some }\end{array}$ & Marker interval $^{a}$ & Physical interval (bp) & $\begin{array}{l}\text { LOD } \\
\text { score }\end{array}$ & $\begin{array}{c}\text { Variance ex- } \\
\text { plained of } \\
\text { total }(\%)\end{array}$ & $\begin{array}{l}\text { Additive } \\
\text { effect }^{b}\end{array}$ & $\begin{array}{c}\text { LOD } \\
\text { threshold }\end{array}$ \\
\hline \multirow{6}{*}{$\begin{array}{l}\text { Brown spot } \\
\text { resistance }^{c}\end{array}$} & 2017 & $q B S R 6-k d$ & 6 & FA0366-FA0370 & $6,088,952-8,196,056$ & 4.9 & 20.3 & -0.7 & 2.8 \\
\hline & 2018 & $q B S R 3.1-k d$ & 3 & FA0201-FA0204 & $6,711,250-8,200,582$ & 3.7 & 6.8 & -0.4 & 2.7 \\
\hline & 2018 & $q B S R 3.2-k d$ & 3 & $\underline{\text { FA0229-FA0233 }}$ & $25,722,435-29,085,775$ & 3.4 & 7.0 & -0.5 & 2.7 \\
\hline & 2018 & $q B S R 7-k d$ & 7 & FA0453-FA0466 & $20,816,371-27,050,785$ & 7.7 & 18.6 & -0.6 & 2.7 \\
\hline & 2019 & qBSR3.1-kd & 3 & FA0201-FA0204 & $6,711,250-8,200,582$ & 2.8 & 6.2 & -0.3 & 2.8 \\
\hline & 2019 & qBSR6-kd & 6 & FA0366- $\underline{\text { FA0370 }}$ & $6,088,952-8,196,056$ & 4.6 & 15.1 & -0.6 & 2.8 \\
\hline \multirow{9}{*}{$\begin{array}{l}\text { Days to } \\
\text { heading }\end{array}$} & 2017 & $q D T H 3-k d$ & 3 & $\underline{\text { FA0233-FA0243 }}$ & $29,085,775-34,611,745$ & 10.1 & 31.7 & 6.3 & 3.2 \\
\hline & 2017 & qDTH6-kd & 6 & $\underline{\text { FA0370-FA0375 }}$ & $8,196,056-12,284,260$ & 9.5 & 18.1 & -4.8 & 3.2 \\
\hline & 2017 & $q D T H 7-k d$ & 7 & FA0453-FA0466 & $20,816,371-27,050,785$ & 6.1 & 11.7 & 3.1 & 3.2 \\
\hline & 2018 & $q D T H 3-k d$ & 3 & FA0233-FA0243 & $29,085,775-34,611,745$ & 10.3 & 31.8 & 6.4 & 3.2 \\
\hline & 2018 & qDTH6-kd & 6 & $\underline{\text { FA0370-FA0375 }}$ & $8,196,056-12,284,260$ & 9.9 & 18.3 & -4.9 & 3.2 \\
\hline & 2018 & qDTH7-kd & 7 & FA0453-FA0466 & $20,816,371-27,050,785$ & 7.0 & 13.2 & 3.4 & 3.2 \\
\hline & 2019 & $q D T H 3-k d$ & 3 & $\underline{\text { FA0233-FA0243 }}$ & $29,085,775-34,611,745$ & 8.8 & 29.4 & 5.2 & 4.0 \\
\hline & 2019 & qDTH6-kd & 6 & $\underline{\text { FA0370-FA0375 }}$ & $8,196,056-12,284,260$ & 6.5 & 12.8 & -3.5 & 4.0 \\
\hline & 2019 & qDTH7-kd & 7 & FA0453- $\underline{\text { FA0466 }}$ & $20,816,371-27,050,785$ & 5.0 & 10.1 & 2.5 & 4.0 \\
\hline
\end{tabular}

${ }^{a}$ The nearest markers are underlined.

${ }^{b}$ Negative values mean that the Dawn allele decreased the trait values.

${ }^{c}$ Brown spot resistance was assessed by disease score: lower scores indicate greater resistance.

\section{Discussion}

Our research group reported that several cultivars from outside Japan are more resistant to BS than Japanese cultivars (Matsumoto et al. 2017a). Because BS is such a problem in Japan, it is important that BS resistance QTLs from these other cultivars be introduced into Japanese rice cultivars. Little is known about major genes conferring immunity to BS, but several cultivars including 'Dawn' have high quantitative resistance (Eruotor 1986, Matsumoto et al. 2016). In this study, we attempted to identify BS resistance QTLs within a BIL population derived from a cross between 'Dawn' and 'Koshihikari'.

Hori et al. (2012) reported that a large number of agronomic traits in rice are correlated with heading date, and in previous studies we observed negative correlations of disease score with heading date (Matsumoto et al. 2017a, $2017 b$ ). In the present study, significant negative correlations between disease score and DTH in the BIL population were observed for three years. It is quite likely that BS resistance in 'Dawn' is associated with pleiotropic effects of genes controlling heading date.

We identified four QTLs for BS resistance on chromosomes 3 (two QTLs), 6, and 7: each QTL explained 6.2\% to $20.3 \%$ of the phenotypic variation (Fig. 3, Table 1). For all four QTLs, the allele from 'Dawn' contributed to BS resistance. One QTL $(q B S R 6-k d)$ explained $15.1 \%-20.3 \%$ of the total phenotypic variation and was linked to $q D T H 6-k d$, at which the 'Dawn' allele decreased DTH. Two QTLs on chromosome 6 associated with BS resistance were reported in previous studies (Dudhare et al. 2008, Katara et al.
2010), but it is difficult to compare their results with ours because their mapping population, DNA markers, and field evaluation of BS differed from ours. One QTL ( $q B S R 3.1$ $k d)$ was unlinked to any of the QTLs for DTH. The above results indicate that $q B S R 6-k d$ and $q B S R 3.1-k d$ are likely to be useful for breeding BS-resistant varieties without delaying heading.

In contrast, two other QTLs for BS (qBSR3.2-kd and $q B S R 7-k d)$ were associated with QTLs for DTH ( $q D T H 3-$ $k d$ and $q D T H 7-k d$ ) at which the 'Dawn' alleles delayed heading. No QTLs related to BS resistance have been reported in these genomic regions. Further studies using near isogenic line for these QTLs and phenotypic methods less confounded by heading date are needed to elucidate the relationship between BS resistance and DTH.

'Koshihikari', the most widely grown cultivar in Japan and the subject of many genetic studies (Kobayashi et al. 2018), was used as the recurrent parent of the BIL population in this study. Thus, the BS resistance QTLs and BILs described here will contribute to improving BS resistance in rice breeding programs in Japan.

\section{Author Contribution Statement}

YO carried out field evaluations and genetic analysis; HS and RM carried out genetic analysis and developed BILs; KM, YN, TY and TO carried out field evaluations; YO, HS and RM wrote the manuscript, with assistance from the other authors. 


\section{Acknowledgments}

The authors thank Y. Nakabayashi, T. Kitagawa, N. Moriguchi, M. Kawaguchi, H. Najime, and M. Kato for their technical assistance. This work was supported by grants from the Ministry of Agriculture, Forestry and Fisheries of Japan (Project for "development of mitigation and adaptation techniques to global warming in the sectors of agriculture, forestry, and fisheries", Rice \#1201 and \#1401).

\section{Literature Cited}

Dudhare, M.S., P.V. Jadhav and S.K. Mishra (2008) Molecular mapping of QTLs for resistance to brown spot disease in rice. J. Plant Dis. Sci. 3: 21-23.

Ebana, K., J. Yonemaru, S. Fukuoka, H. Iwata, H. Kanamori, N. Namiki, H. Nagasaki and M. Yano (2010) Genetic structure revealed by a whole-genome single-nucleotide polymorphism survey of diverse accessions of cultivated Asian rice (Oryza sativa L.). Breed. Sci. 60: 390-397.

Eruotor, P.G. (1986) Varietal reaction of rice to isolates of Cochliobolus miyabeanus. Indian Phytopath. 39: 62-64.

Hori, K., T. Kataoka, K. Miura, M. Yamaguchi, N. Saka, T. Nakahara, Y. Sunohara, K. Ebana and M. Yano (2012) Variation in heading date conceals quantitative trait loci for other traits of importance in breeding selection of rice. Breed. Sci. 62: 223-234.

Hori, T., R. Saito, H. Sakuma, K. Ono, K. Takahashi, S. Matsuzawa, Y. Yamaguchi and K. Ogata (2019) Some factors causing the outbreaks of brown spot in rice. J. of the Niigata Agri. Res. Inst. 17: 51-54.

Japan Plant Protection Association (2019) Epidemic and controlling areas in 2018. In: Japan Plant Protection Association (ed.) Catalogue of agricultural chemicals, Tokyo, pp. 602-603.

Katara, J.L., H. Sonah, R.K. Deshmukh, R. Chaurasia and A.S. Kotasthane (2010) Molecular analysis of QTLs associated with resistance to brown spot in rice (Oryza sativa L.). Indian J. Genet. Plant Breed. 70: 17-21.

Kihara, J. and T. Kumagai (1994) Ecotypes of the fungus Bipolaris oryzae with various responses of the mycochrome system. Physiol. Plant. 92: 689-695.

Kobayashi, A., K. Hori, T. Yamamoto and M. Yano (2018) Koshihikari: a premium short-grain rice cultivar-its expansion and breeding in Japan. Rice (N Y) 11: 15.

Lander, E.S., P. Green, J. Abrahamson, A. Barlow, M.J. Daly, S.E.
Lincoln and L.A. Newburg (1987) MAPMAKER: an interactive computer package for constructing primary genetic linkage maps of experimental and natural populations. Genomics 1: 174-181.

Liu, R.H. and J.L. Meng (2003) MapDraw: a Microsoft Excel macro for drawing genetic linkage maps based on given genetic linkage data. Hereditas 25: 317-321.

Matsumoto, K., H. Sato, C. Ota, S. Seta, T. Yamakawa, H. Suzuki and Y. Nakayama (2016) A new method for evaluating field resistance to brown spot in rice. Breed. Res. 18: 103-111 (in Japanese with English summary).

Matsumoto, K., S. Seta, C. Ota, T. Ohno, Y. Ota, Y. Nakayama, T. Yamakawa and H. Sato (2017a) Search for genetic resources resistant to brown spot in NIAS core collections of Japanese rice landraces and world rice. Breed. Res. 19: 155-163 (in Japanese).

Matsumoto, K., Y. Ota, S. Seta, Y. Nakayama, T. Ohno, R. Mizobuchi and H. Sato (2017b) Identification of QTLs for rice brown spot resistance in backcross inbred lines derived from a cross between Koshihikari and CH45. Breed. Sci. 67: 540-543.

McCouch, S.R., CGSNL (Committee on Gene Symbolization, Nomenclature and Linkage, Rice Genetics Cooperative) (2008) Gene nomenclature system for rice. Rice (N Y) 1: 72-84.

Mizobuchi, R., S. Fukuoka, S. Tsushima, M. Yano and H. Sato (2016) QTLs for resistance to major rice diseases exacerbated by global warming: brown spot, bacterial seedling rot, and bacterial grain rot. Rice (N Y) 9: 23.

Murray, M.G. and W.F. Thompson (1980) Rapid isolation of highmolecular-weight plant DNA. Nucleic Acids Res. 8: 4321-4325.

Padmanabhan, S.Y. (1973) The great Bengal famine. Annu. Rev. Phytopathol. 11: 11-24.

Sato, H., I. Ando, H. Hirabayashi, Y. Takeuchi, S. Arase, J. Kihara. H. Kato and H. Nemoto (2008) QTL analysis of brown spot resistance in rice (Oryza sativa L.). Breed. Sci. 58: 93-96.

Sato, H., K. Matsumoto, C. Ota, T. Yamakawa, J. Kihara and R. Mizobuchi (2015) Confirming a major QTL and finding additional loci responsible for field resistance to brown spot (Bipolaris oryzae) in rice. Breed. Sci. 65: 170-175.

Sunder, S., R. Singh and R. Agarwal (2014) Brown spot of rice: an overview. Indian Phytopath. 67: 201-215.

Wang, S., C.J. Basten and Z.B. Zeng (2006) Windows QTL Cartographer 2.5. Department of Statistics, North Carolina State University, Raleigh, NC. https://brcwebportal.cos.ncsu.edu/qtlcart/ WQTLCart.htmg. Cited 23 Oct. 2006.

Yamaguchi, Y., K. Nakano and R. Saito (2007) An outbreak of rice brown spot at Niigata prefecture Kaetsu district in 2005 and 2006. The Association for Plant Protection of Hokuriku 59: 4. 